\title{
The Lancet: la fin de l'objectivité dans la recherche scientifique
}

\author{
B. Ferroni
}

Dans son édition du 27 août 2005, The Lancet a publié une méta-analyse du Professeur Egger de l'Institut de Médecine Sociale et Préventive de l'Université de Berne portant sur 110 études en double aveugle en homéopathie comparées avec 110 études en médecine conventionnelle [1]. Une vingtaine de réactions et analyses critiques rédigés par des chercheurs de renom ont été adressées au Lancet dans les 15 jours qui ont suivi la publication. Pas une seule d'entre elles n'a été publiée à ce jour. Cette absence d'objectivité dans le débat scientifique surprend, d'autant plus qu'elle émane d'un journal très en vue dans le monde médical. C'est la raison pour laquelle une clarification des faits s'impose.

La méta-analyse d'Egger, Shang et al. prétend ne pas avoir pu démontrer d'effet spécifique de l'homéopathie dans l'échantillonnage des travaux en double aveugle analysés. Mais à la lecture attentive de cette publication, l'on est surpris de constater qu'en fait l'homéopathie montre un effet positif, tout comme l'allopathie, dans 102 des petites et moyennes études analysées; ce n'est que dans un échantillon sélectif de 8 (huit!) grandes études, qui d'ailleurs manquent totalement de représentativité et de validité en homéopathie, que M. Egger démontre un effet moindre que seulement les 6 études allopathiques comparées. C'est l'extrapolation des résultats de ces 8 études qui amène les auteurs de la publication du Lancet à leur conclusion que les effets bénéfiques observés au cours des traitements homéopathiques seraient «compatibles avec l'hypothèse du placebo».

Cette conclusion d'Egger/Shang est en flagrante opposition avec les résultats du «Health Technology Assessement» sur l'homéopathie élaboré dans le cadre des recherches PEK en Suisse ces dernières années et qui va être publié prochainement.

Cette conclusion contredit totalement l'expérience de milliers de médecins homéopathes à travers le monde.

Cette conclusion est de surplus en totale opposition avec les résultats de 20 des 22 métaanalyses et revues systématiques publiées sur les recherches cliniques en homéopathie (cf ciaprès).
Mais tous ces éléments n'ont pas empêché les éditorialistes du Lancet de reprendre avec fracas les conclusions de cette étude en annonçant la «fin de l'homéopathie» [2]. Plus moralisateur que scientifique, le Lancet cherche à enterrer une discussion dérangeante sur une méthode thérapeutique qui a montré des résultats évidents, mais dont la preuve finale de l'efficacité n'est pas encore définitivement apportée aux yeux du courant académique majoritaire de nos jours.

Voici ce que montre l'analyse critique de l'étude ISPM d'Egger/Shang:

- Cette étude manque de transparence et de reproductibilité. Les travaux sur lesquels sont basées les conclusions de cette étude ne sont pas référencées.

- La sélection des études est biaisée au départ et toutes les études n'ont pas été prises en compte. Il existe entre 300-400 travaux en double aveugle publiés évaluant les effets des traitements homéopathiques. Les 110 études retenues par Egger ne représentent donc de loin pas la «quasi-totalité des études», comme il l'écrit, mais un échantillonnage non représentatif de ces dernières, violant ainsi les règles et les standards de la Cochrane Collaboration

- Environ 3/4 des 110 travaux homéopathiques examinés montrent des résultats positifs, tout comme plusieurs grandes revues et méta-analyses publiées durant ces dernières années (pour n'en mentionner que les plus importantes: [3-9]). En aucune façon, M. Egger ne discute ni ne mentionne ce fait troublant.

- Seules huit études (7\%[!]) sur les 110 évaluées sont à la base des conclusions des auteurs. Les 93\% restantes ont été sous-pondérées, en raison de leur taille et de leur «faible validité interne».

- La validité interne (c.-à-d. la qualité méthodologique d'une étude) des travaux analysés est le seul critère retenu pour leur sélection tandis que la validité externe (= la représentativité d'une étude dans la pratique de la thérapie analysée) a été totalement négligée; seuls 18 travaux sur 110 concernent l'homéopathie classique. C'est dire que les études analysées ne reflètent en aucune manière 
l'homéopathie, telle qu'elle est concrètement pratiquée dans les cabinets dans le cadre de la médecine de premier recours et telle qu'elle a été évaluée par le PEK.

- Toutes ces critiques fondamentales qui privent l'étude de l'ISPM de toute crédibilité étaient connues depuis deux ans par ses auteurs, et par M. Egger tout particulièrement. Ils ont tout simplement refusé d'en tenir compte, ne serait-ce qu'au niveau de la discussion de leurs résultats. L'on se demande où est l'objectivité scientifique?

Rappelons que cette méta-analyse faisait partie du Programme d'évaluation des médecines complémentaires PEK qui investiguait l'homéopathie classique (uniciste) en Suisse. Une discussion contradictoire de cette «étude ISPM» a eu lieu entre M. Egger et les experts de la Société suisse des médecins homéopathes en date du 3 juin 2004 déjà. Une prise de position critique est publiée, à côté d'autres documents, sur internet [10].

Le débat scientifique sur l'homéopathie reste donc ouvert, n'en déplaise à certains. Il est surprenant de constater que l'objectivité et la rigueur scientifique du Lancet n'ont pas incité les responsables de ce journal à comparer ces résultats contradictoires et à en discuter les tenants et aboutissants. Ceci d'autant plus qu'en même temps que l'étude de l'ISPM, le Lancet a reçu une demande de publication d'un autre travail de recherche émanant de la même Université de Berne, démontrant clairement dans un setting en double aveugle l'efficacité de l'homéopathie dans le traitement d'enfants hyperactifs [11]. Comment se fait-il que ces résultats contradictoires n'aient pas nourri la discussion académique, mais aient simplement été passés sous silence par les éditeurs tout autant que par les auteurs? Où reste la crédibilité de la recherche scientifique? Qu'est devenue la fiabilité du Lancet? Le but recherché, est-il de faire taire des voix dissonantes, alors que la recherche fondamentale continue à lever - timidement, il est vrai, mais par petits pas tout de même $[12,13]$ le voile sur l'effet de ces mystérieuses dilutions ultra-moléculaires au mécanisme d'action inconnu? Dans sa recherche d'objectivité, la science est peut-être bien conseillée de se rappeler la phrase de Claude Bernard, père de notre médecine expérimentale: «Quand le fait que l’on rencontre ne s'accorde pas avec une théorie régnante, il faut accepter le fait et abandonner la théorie.»

\section{Références}

1 Shang A, Huwiler-Müntener K, Nartey L, et al. Are the clinical effects of homoeopathy placebo effects? Comparative study of placebo-controlled trials of homoeopathy and allopathy. Lancet 2005;366:726-32.

2 Editorial. Lancet 2005;366:690.

3 Kleijnen J, et al. Clinical trials of homoeopathy. BMJ 1991;302:316-23.

4 Boissel J. Critical Literature Review on the effectiveness of Homeopathy: Overview over data from homeopathic medicine trials. In: Commission of the European communities (ed.). Homeopathic Medicine Research Group. Brussels: Commission of the European communities; 1996. p. 196-210.

5 Linde K, et al. Are the effects of homeopathy all placebo effects? A meta-analysis of randomised, placebo controlled trials. Lancet 1997;350:834-43.

6 Cucherat M, et al. Evidence of clinical efficacy of homeopathy. A meta-analysis of clinical trials. HMRAG Homeopathic Medicines Research Advisory Group. Eur J Clin Pharmacol 2000;56:27-33.

7 Wein C. Qualitätsaspekt klinischer Studien zur Homöopathie. Essen: KVC; 2002.

8 Mathie RT. The research evidence base for homeopathy: a fresh assessment of the literature. Homeopathy 2003;92(2):84-91.

9 Dean M. Out of step with the Lancet homeopathy meta-analysis: more objections than objectivity? J Alternative Complementary Med 1998; 4(4):389-98.

10 Stellungnahme des Schweizerischen Vereins homöopathischer Ärztinnen und Ärzte SVHA zur Homöopathiestudie des Instituts für Sozial- und Präventivmedizin ISPM Bern («Egger-Studie»). Bern: SSMH; 2005. www.ssmh.ch.

11 Frei H, et al. Homeopathic treatment of children with attention deficit hyperactive disorder: a randomised, double blind, placebo controlled crossover trial. http://dx.doi.org/10.1007/ s00431-005-1735-7. 27.7.2005.

12 Rey L. Thermoluminesence of ultra-high dilutions of lithium chloride and sodium chloride. Physica A 2003;323:67-74.

13 Suess W, et al. Mechanism of the transfer of the «therapeutically active ingredient» (TAI) from a homeopathic liquid dilution to a solid substance. In: Abstracts of the $60^{\text {th }}$ Congress of the Liga Medicorum Homeopathica internationalis, Berlin 2005. AHZ 2005;250:37 (suess@rz.uni-leipzig.de) 